\title{
Semi-Omnidirectional Dual-Polarized Wideband Multiport Antennas for MIMO Applications in Random-LOS and RIMP
}

\author{
Sadegh Mansouri Moghaddam ${ }^{1}$, Andrés Alayón Glazunov ${ }^{1}$, Jian Yang ${ }^{1}$, Mattias Gustafsson ${ }^{2}$ \\ ${ }^{1}$ Department of Signals and Systems, Chalmers University of Technology, Gothenburg, Sweden, sadegh.mansouri@chalmers.se \\ ${ }^{2}$ Huawei Technologies, Sweden AB.
}

\begin{abstract}
We present two configurations of multiport antennas for Multiple-input Multiple-output (MIMO) application. The configurations are comprised of three and four dual-polarized selfgrounded bowtie antenna as the element, respectively. The MIMO performance of both antennas is evaluated in Random Lineof-Sight (Random-LOS) and Rich Isotropic Multipath (RIMP) channel models as two edge propagation environments. Both configurations provide $360^{\circ}$ azimuth and $120^{\circ}$ elevation angular coverage in Random-LOS and full sphere coverage in RIMP. Using digital threshold receiver model and Zero-Forcing receiver, the performances of both configurations are evaluated in terms of Probability of Detection (PoD) and MIMO multiplexing efficiency calculated at $95 \%$ PoD level. The simulated results show a good performance for both structures in two edge environments, which can be concluded as a good performance in a real life situation.

Index Terms-MIMO, Random-LOS, RIMP, Bowtie, PoD.
\end{abstract}

\section{INTRODUCTION}

Making use of multiple port antennas at both the transmitter and the receiver sides, known as MIMO (Multiple Input Multiple Output), has increased the channel capacity and the reliability of the wireless systems without increasing the input power or frequency bandwidth. The antenna diversity and spatial multiplexing can be performed to remove timevarying fading of the receiving single bitstream and to transmit multiple data-streams, respectively [1]. Indoor MIMO communication systems can make use of dual-polarized antennas with omnidirectional radiation function to improve the system performance [2]. Several dual-polarized omnidirectional antenna are introduced in literature [3], [4]. In [5] a wideband flat Eleven antenna was designed as MIMO antenna for micro base station, however with a limited angular coverage. Due to space limitation, achieving an omnidirectional radiation characteristics while providing dual polarization with high cross polarization discrimination and port isolation over a wide frequency bandwidth is quite a challenge.

On the other hand, taking advantage of multiple antennas are directly related to the channel model in which we evaluate the performance of our antennas. For applications such as micro base stations, the user and the base station antenna can be connected to each other either by reflected and scattered field components, i.e. Rich Isotropic Multipath (RIMP) or by one significant component, i.e. Line of Sight (LOS). This LOS component will be random due to the user's randomness in location, i.e. Angle of Arrival (AoA), and orientation, i.e. polarization. Thus, in order to take into account these stochastic characteristics, the term Random-LOS was introduced in [6]. In [7], both RIMP and Random-LOS are introduced as the reference (or edge) environments and a real life scenario would always be somewhere between the two edges. A real life hypothesis was stated in [8] as "if a wireless device works well in both RIMP and Random-LOS, it will also work well in any real-life environment".

In this paper, we present two structures comprised of bowtie elements [9]-[11] to resemble a wideband dual-polarized omnidirectional antennas for MIMO application. The element is designed to have a good performance in an embedded configuration. To evaluate the MIMO performance of both structures, the Probability of Detection (PoD) curves are plotted and MIMO multiplexing efficiencies are calculated. By the real life hypothesis, the MIMO performance evaluation is done in both Random-LOS and RIMP as two complementary reference propagation environments.

\section{Antennas StRuctures}

Two different configurations are show in Fig. 1. The element is presented based on the design in [12] and modified for the current application. Each element is comprised of four selfgrounded petals bent in discrete angles, rise from separate feed points at the surface of a Teflon block in the middle of the antenna. Two opposite petals are differentially excited to provide a dual-polarized (horizontal and vertical) element. The first configuration is consist of three of these bowtie elements referred to the triple structure. In order to realize the same boundaries for both horizontal and vertical polarizations, an extended wall is added to each sides of each elements. This results in an almost the same radiation characteristics for both polarizations. The second configuration is consist of four backto-back bowties introduced as quadruple structure. In this case, an extra plate was used to connect the extended walls in order to improve the radiation pattern at lower frequencies. The maximum dimension of whole antenna is $134 \mathrm{~mm}$ and 171 $\mathrm{mm}$ for the triple and quadruple structure, respectively. 


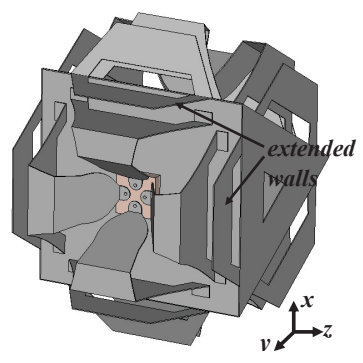

(a)

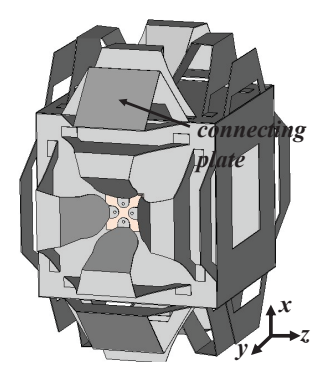

(c)

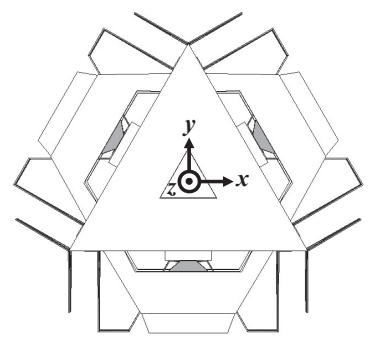

(b)

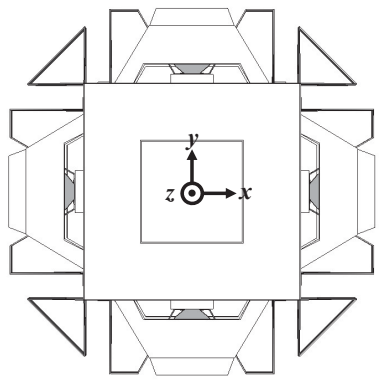

(d)
Fig. 1. The geometry of the proposed configuration. a) $3 d$ and b) side view of the triple structure and c) $3 \mathrm{~d}$ and d) side view of the quadruple structure.

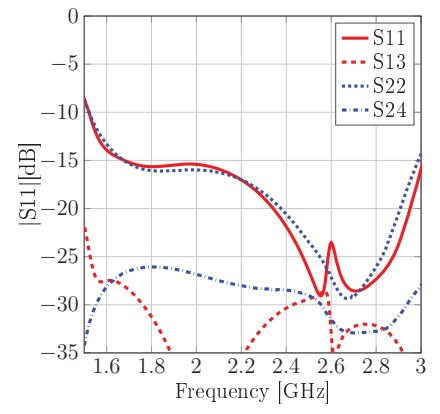

(a)

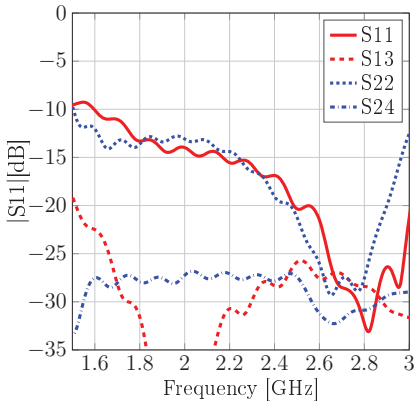

(b)
Fig. 2. The scattering parameters of embedded bowtie element for both polarizations for a) triple and b) quadruple structure.

The simulated scattering parameters of the embedded elements in both structures are shown in Fig. 2. Port 1 and Port 2 represents the horizontal and vertical polarizations, respectively. Port 3 and 4 are the adjacent ports of the neighboring element with the same polarization as port 1 and port 2, respectively. To clarify this, the locations of the ports in quadruple configuration are illustrated in Fig. 3. As can be seen, The reflection coefficient for both polarizations remains below $-10 \mathrm{~dB}$ over the frequency band of $1.5-3 \mathrm{GHz}$. The isolation is fairly better than $25 \mathrm{~dB}$ over the frequency band of desire for both polarizations. The coupling between the two orthogonally polarized differentially excited ports is negligible and therefore it is not shown. In addition, the directivity and total radiation efficiency of the embedded element in both structures and for both polarizatins are plotted in Fig. 4. The corresponding directivities are within $5.5-8 \mathrm{dBi}$ and the total radiation efficincies are almost higher than $90 \%(-0.4 \mathrm{~dB})$ for all the cases within $1.5-3 \mathrm{GHz}$.

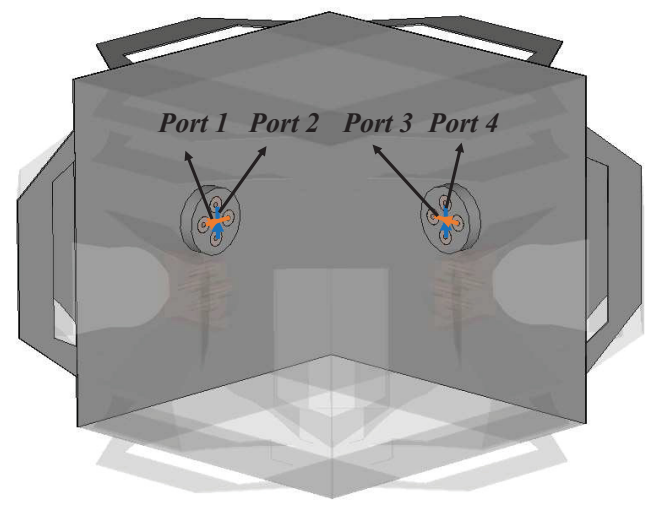

Fig. 3. The locations of ports in quadruple configurations.

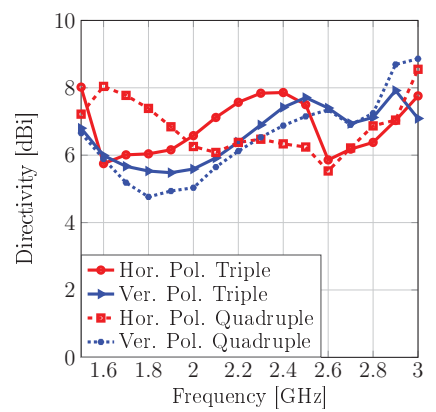

(a)

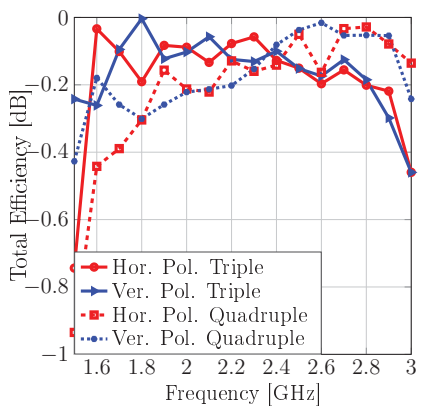

(b)
Fig. 4. a) Directivity (dBi) and Total efficiency (dB) of embedded bowtie element for both polarizations and for both structures.

\section{MIMO PERFORMANCE}

We use the digital threshold receiver model [13] to provide the probability of detection $(\mathrm{PoD})$ of data-streams representing the relative throughput. The Zero-Forcing algorithm in receiver side is used to separate these bitstreams as the independent information. The PoD for detection of multiple bitstreams is defined as the PoD of detecting the weakest bitstream channel in each realization of the channel matrix.

\section{A. In Random-LOS}

The maximum available bitsreams in a space-limited scenario in Random-LOS propagation environment is equal to two, corresponding to two orthogonal polarizations. We restrict our desired coverage to $360^{\circ}$ in azimuth and $120^{\circ}$ in elevation angle. Also, linear horizontal and vertical polarizations are considered as two orthogonal data-streams subchannels. To demonstrate the Random-LOS scenario, two incoming waves have arbitrary orthogonal polarization coming from the same AoA, uniformely distributed in the desired coverage. A 2port antenna with $100 \%$ efficiency and perfect orthogonally polarized far-field function and equal gain over the entire aimed coverage is assumed as a reference. The corresponding PoD curves for both structures at three different frequencies are shown in Fig. 5. The MIMO multiplexing efficiency can be defined as the difference between the antenna and the 


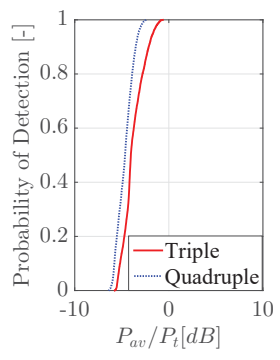

(a)

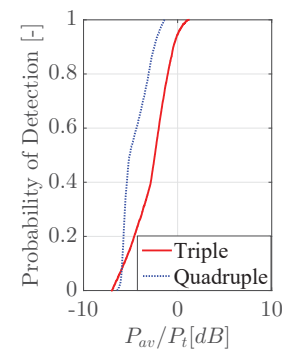

(b)

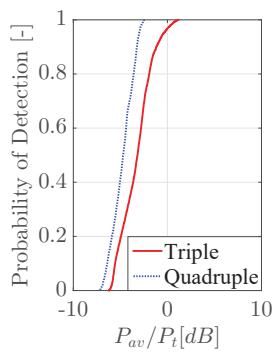

(c)
Fig. 5. Probability of Detection (PoD) of two bitstreams in Random-LOS within the desired coverage at a) $1.7 \mathrm{GHz}$, b) $2.2 \mathrm{GHz}$ and c) $2.7 \mathrm{GHz}$ for both structures.

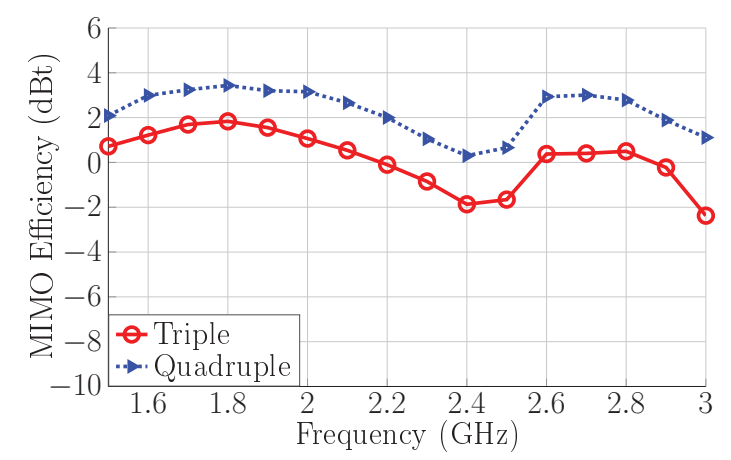

Fig. 6. MIMO multiplexing efficiency (in $\mathrm{dBt}$ ) in Random-LOS within the desired coverage for both structures.

reference at 95\% PoD level. In Fig. 6 we see that the MIMO multiplexing efficiency can be higher than $0 \mathrm{~dB}$. This arises from the MRC diversity gain achieved by using multiple ports. Also we can observe that this value is between 1 to $2 \mathrm{~dB}$ higher for the quadruple structure which implies a higher diversity gain for this case.

\section{B. In RIMP}

By plotting the corresponding PoD curves in RIMP, using digital threshold receiver model and Zero-Forcing receiver, the MIMO multiplexing efficiency in dBiid can be calculated at $95 \%$ PoD level. The i.i.d. (independent and identically distributed) refers to ports with $100 \%$ embedded efficiency and no correlation. Fig. 7 shows the MIMO multiplexing efficiencies of both structure for different MIMO systems. We can see that the performance is directly proportional to the embedded efficiencies of the elements. This is because of the low electromagnetically correlation of ports in terms of polarization and radiation direction of their far-field functions. We also see that the MIMO multiplexing efficiency is almost independent from the number of antennas. This implies the low pattern correlation of elements even for higher order MIMO configurations.

\section{CONCLUSiON}

Two structures was presented to achieve desirable MIMO performance. The MIMO performances of both structures

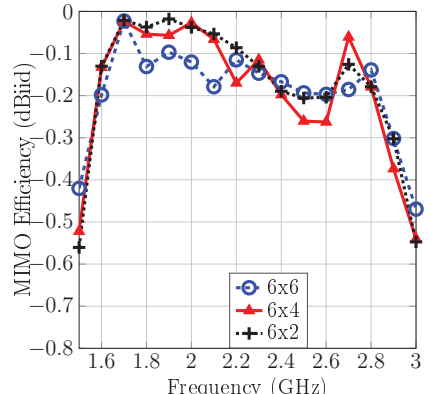

(a)

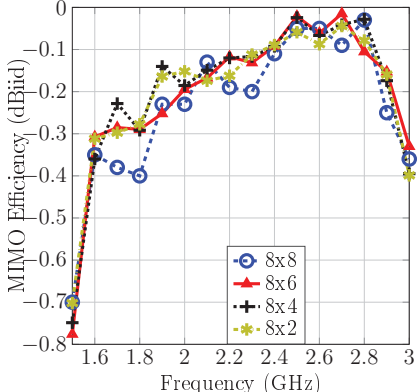

(b)
Fig. 7. MIMO multiplexing efficiency (in dBiid) in RIMP for both structures in different MIMO systems.

was evaluated in Random-LOS and RIMP channel model as two references for the real life propagation environment. In Random-LOS, the MIMO efficiencies (in $\mathrm{dBt}$ ) of both structures remained fairly constant over the entire frequency band of interest. This value was around $2 \mathrm{~dB}$ higher for quadruple structure originating from the higher diversity gain derived from using higher number of the ports, sacrificing the overall volume of the antenna. Because of the low correlation of the voltages on the ports, the MIMO efficiency (in dBiid) in RIMP follows the total radiation efficiency of the embedded elements. This was achieved by the low polarization coupling and different radiation direction of the antenna ports.

\section{ACKNOWLEDGMENT}

The present work has been supported by the Swedish Governmental Agency for Innovation Systems (VINNOVA) within the VINN Excellence Center Chase at Chalmers, and by the same VINNOVA with a project on MIMO hardware within the Innovative ICT 2013 program.

\section{REFERENCES}

[1] P.-S. Kildal, X. Chen, M. Gustafsson, and Z. Shen, "MIMO characterization on system level of $5 \mathrm{G}$ microbase stations subject to randomness in LOS," Access, IEEE, vol. 2, pp. 1064-1077, 2014.

[2] D. Guo, K. He, Y. Zhang, and M. Song, "A multiband dual-polarized omnidirectional antenna for indoor wireless communication systems."

[3] X. Quan and R. Li, "A broadband dual-polarized omnidirectional antenna for base stations," IEEE Transactions on Antennas and Propagation, vol. 61, no. 2, pp. 943-947, 2013.

[4] Y. Li, Z. Zhang, J. Zheng, and Z. Feng, "Compact azimuthal omnidirectional dual-polarized antenna using highly isolated colocated slots," IEEE Transactions on Antennas and Propagation, vol. 60, no. 9, pp 4037-4045, 2012.

[5] A. Razavi, W. Yu, J. Yang, and A. A. Glazunov, "Design of a planar eleven antenna for optimal mimo performance as a wideband micro base-station antenna," Submitted to IEEE Transactions on Antennas and Propagation, September 2016.

[6] P.-S. Kildal, "Rethinking the wireless channel for OTA testing and network optimization by including user statistics: RIMP, pure-LOS, throughput and detection probability," in ISAP 2013, Nanjing, 2013.

[7] P.-S. Kildal, C. Orlenius, and J. Carlsson, "Ota testing in multipath of antennas and wireless devices with mimo and ofdm," Proceedings of the IEEE, vol. 100, no. 7, pp. 2145-2157, 2012.

[8] P.-S. Kildal and J. Carlsson, "New approach to ota testing: Rimp and pure-los reference environments \& a hypothesis," in Antennas and Propagation (EuCAP), 2013 7th European Conference on. IEEE, 2013, pp. 315-318. 
[9] J. Yang and A. Kishk, "A novel low-profile compact directional ultrawideband antenna: the self-grounded bow-tie antenna," IEEE Transactions on Antennas and Propagation, vol. 60, no. 3, pp. 1214-1220, 2012.

[10] H. Raza, A. Hussain, J. Yang, and P.-S. Kildal, "Wideband compact 4 port dual polarized self-grounded bowtie antenna," IEEE Transactions on Antennas and Propagation, vol. 62, no. 9, pp. 4468-4473, 2014

[11] A. Al-Rawi, A. Hussain, J. Yang, M. Franzén, C. Orlenius, and A. A Kishk, "A new compact wideband mimo antennaâĂ $\breve{T}$ the double-sided tapered self-grounded monopole array," IEEE Transactions on Antennas and Propagation, vol. 62, no. 6, pp. 3365-3369, 2014.

[12] S. M. Moghaddam, P.-S. Kildal, A. A. Glazunov, and J. Yang, "Designing a dual-polarized octave bandwidth bowtie antenna for a linear array."

[13] P.-S. Kildal, A. Hussain, X. Chen, C. Orlenius, A. Skårbratt, J. Åsberg, T. Svensson, and T. Eriksson, "Threshold receiver model for throughput of wireless devices with MIMO and frequency diversity measured in reverberation chamber," Antennas and Wireless Propagation Letters, IEEE, vol. 10, pp. 1201-1204, 2011 